\title{
CURVATURE OF QUANTUM RINGS
}

\author{
Edmond Jonckheere ${ }^{1}$, Frank C. Langbein ${ }^{2}$ and Sophie G. Schirmer ${ }^{3}$ \\ ${ }^{1}$ USC Center for Quantum Information Science \& Technology, Los Angeles, CA 90089, USA \\ ${ }^{2}$ School of Computer Science and Informatics, Cardiff University, 5 The Parade, CF24 3AA, UK \\ ${ }^{3}$ College of Science (Physics), Swansea University, Singleton Park, Swansea, SA6 7JY, UK
}

\begin{abstract}
We develop a geometric approach to spin networks with Heisenberg or XX coupling. Geometry is acquired by defining a distance on the discrete set of spins. The key feature of the geometry of such networks is their Gauss curvature $\kappa$, viewed here as the ability to isometrically embed the chain in the standard Riemannian manifold of curvature $\kappa$. Here we focus on spin rings. Even though their visual geometry is trivial, it turns out that the geometry they acquire from the quantum mechanical distance is far from trivial.
\end{abstract}

Index Terms - Spin chains, coarse geometry, curvature, Riemannian spaces, Feynman path integral.

\section{INTRODUCTION}

We consider 1-dimensional arrays of $N$ spins arranged in a ring structure with either Heisenberg or XX interaction specified by the Hamiltonian

$$
\begin{aligned}
H= & \sum_{i=1}^{N-1} J_{i, i+1}\left(\sigma_{i}^{x} \sigma_{i+1}^{x}+\sigma_{i}^{y} \sigma_{i+1}^{y}+\epsilon \sigma_{i}^{z} \sigma_{i+1}^{z}\right) \\
& +J_{N, 1}\left(\sigma_{N}^{x} \sigma_{1}^{x}+\sigma_{N}^{y} \sigma_{1}^{y}+\epsilon \sigma_{N}^{z} \sigma_{1}^{z}\right)
\end{aligned}
$$

where $\epsilon=0$ for XX coupling and $\epsilon=1$ for Heisenberg coupling, which we shall denote by $H_{\mathrm{H}}$ and $H_{\mathrm{XX}}$ in the following. The term $J_{N, 1}(\ldots)$ represents the coupling energy between the two ends, spin \#1 and \#N, of the linear array, closing the ring. The factor $\sigma_{i}^{x, y, z}$ is the Pauli matrix along the $x, y$, or $z$ direction of spin $\# i$ in the array, i.e.,

$$
\sigma_{i}^{x, y, z}=I_{2 \times 2} \otimes \ldots \otimes I_{2 \times 2} \otimes \sigma^{x, y, z} \otimes I_{2 \times 2} \otimes \ldots \otimes I_{2 \times 2}
$$

where the factor $\sigma^{x, y, z}$ occupies the $i$ th position among the $N$ factors and $\sigma^{x, y, z}$ is either of the single spin Pauli operators

$$
\sigma^{x}=\left(\begin{array}{cc}
0 & 1 \\
1 & 0
\end{array}\right), \quad \sigma^{y}=\left(\begin{array}{cc}
0 & -\imath \\
\imath & 0
\end{array}\right), \quad \sigma^{z}=\left(\begin{array}{cc}
1 & 0 \\
0 & -1
\end{array}\right)
$$

E. Jonckheere was supported by the One Wales Research Institute for Visual Computing (RIVIC), the US National Science Foundation under Grant NetSE 1017881, and ARO MURI grant W911NF-11-1-0268.

F.C. Langbein was supported by One Wales Research Institute for Visual Computing (RIVIC).

S.G. Schirmer was supported by EPSRC ARF Grant EP/D07192X/1 and Hitachi.
$J_{i, i+1}$ denotes the strength of the coupling between spin $\# i$ and spin $\#(i+1)$ and is inversely proportional to the cubic power of the physical distance between spin \# $i$ and spin $\#(i+1)$, here taken to be uniform (homogeneous arrays). The main point of this paper is that the simplicity of the geometry given by the physical distance between spins hides a much more complicated geometry that the network acquires via a quantum mechanically relevant distance. The latter is related to be the (maximum) probability of transmission of an excitation from one spin to another. Here we restrict our attention to the single excitation subspace, i.e., it is assumed that the total number of excitations in the network is one. An excitation is transmitted from one spin and read out from any other spin.

Through the quantum mechanical distance, the spin network acquires a geometry completely different from the simple geometry of the physical arrangement of the spins. For example, a ring made up of an arbitrary large but even number $N$ of spins becomes a regular $[N / 2]$-simplex for the quantum mechanical distance. Beyond this simple illustrative example, here by geometry we mean curvature, which can be defined for either Riemannian or non-Riemannian spaces [5]. For metric possibly non-Riemannian spaces, curvature can be defined via the Gromov $\delta$ or the scaled Gromov $\delta$ (see [4, 3] for various definitions of $\delta$ and [6, 10, 7] for various definitions of the scaled $\delta$.) Recall that the Gromov $\delta$ measures the "fatness" of the geodesic triangles, with the idea that "thin" triangles are symptomatic of negative curvature whereas "fat" triangles are symptomatic of positive curvature. The Gromov $\delta$ approach to curvature of spins in various geometrical arrangements was done in [8]. Here we basically perform the same analysis but remain closer to the traditional Riemannian approach and focus on ring structures instead of linear chains. More specifically, we investigate whether there exists an isometric embedding $(\mathcal{V}, d) \hookrightarrow \mathbb{M}_{\kappa}^{r}$, where $(\mathcal{V}, d)$ is the metric space of the spins endowed with their quantum mechanical distance and $\mathbb{M}_{\kappa}^{r}$ is the standard $r$-dimensional Riemannian space of uniform curvature $\kappa$.

\section{THE QUEST FOR A DISTANCE}

Let $|i\rangle$ be the quantum state where the excitation is on spin $\# i$. The quantum mechanical probability of transition from state 
$|i\rangle$ at time 0 to state $|j\rangle$ at time $t$ is given by

$$
p(|i, 0\rangle,|j, t\rangle)=\left|\left\langle i\left|e^{-\imath H t}\right| j\right\rangle\right|^{2}
$$

Recall that this formula is a corollary of the Feynman path integral [9, 11].

In order to derive a metric on the vertex set $\mathcal{V}=\{|i\rangle: i=1, \ldots, N\}$ from the probability data, we inspire ourselves from a closely related situation in sensor networks. In sensor networks, $\mathcal{V}$ is the set of sensors and a packet reception rate $\operatorname{PRR}(i, j)$ is defined as the probability of successful transmission of the packets from sensor \# $i$ to sensor $\# j$. Then as shown in [1] a useful "distance" is given by $d(i, j)=-\log \operatorname{PRR}(i, j)$. Should there be a violation of the triangle inequality, say, $d(i, j)>d(i, k)+d(k, j)$, then the distance between $i$ and $j$ is redefined as $d(i, k)+d(k, j)$.

We follow the same path here, with the warning that packet transmission from $\# i$ to $\# j$ follows one wireless link, whereas quantum mechanical transition from $|i\rangle$ to $|j\rangle$ follows many paths. Following the approach of [1], we could define a "distance" as $-\log p(|i, 0\rangle,|j, t\rangle)$, but this would make the distance time-dependent. To remove the dependency on the time, define $\Pi_{k}$ to be the projector onto the $k$ th eigenspace of the Hamiltonian $H=\sum_{k} \lambda_{k} \Pi_{k}$ and let $k=1, \ldots, N$ correspond to the first excitation subspace $\mathcal{H}_{1}$. Then, as in [8], we define maximum transition probability also referred to as Information Transfer Capacity:

$$
p_{\max }(|i\rangle,|j\rangle):=\left|\sum_{k=1}^{N}\right|\left\langle i\left|\Pi_{k}\right| j\right\rangle||^{2} \geq p(|i, 0\rangle,|j, t\rangle)
$$

Furthermore, as proved in [8], under the condition that $\lambda_{k} / \pi$ : $k=1, \ldots, N$ are rationally independent, the maximum transition probability can be reached: $\sup _{t \geq 0} p(|i, 0\rangle,|j, t\rangle)=$ $p_{\max }(|i\rangle,|j\rangle)$. Although we cannot in general expect $-\log p_{\max }(|i\rangle,|j\rangle)$ to satisfy the usual requirements for a distance, we show that this is the case for certain types of networks, which allows us to study their geometry and curvature with regard to this metric.

\section{UNIFORM SPIN RINGS}

Unlike for linear chains, the single excitation subspace Hamiltonians for rings with uniform XX and Heisenberg coupling differ only by a multiple of the identity, which does not affect the distance. Hence, the analysis is the same for both of these physically relevant cases. The properties of $-\log p_{\max }(|i\rangle,|j\rangle)$ and the behavior of the distance with $N$ are given by the following:

Theorem 1 For a quantum ring $\mathcal{R}_{N}$ of $N$ uniformly distributed spins with XX or Heisenberg couplings, $d_{N}(i, j):=$ $-\log p_{\max }(|i\rangle,|j\rangle)$ has the following properties:

1. For $N$ odd $\left(\mathcal{R}_{N}, d_{N}\right)$ is a metric space.
2. For $N$ even $\left(\mathcal{R}_{N}, d_{N}\right)$ is a semi-metric space that becomes metric after antipodal point identification.

3. If $N=p$ or $N=2 p$, where $p$ is a prime number, then the distances on the space of equivalence classes of spins are uniform, i.e., $d_{N}(i, j)=c_{N}$ for $i \neq j$. Otherwise, the distances are non-uniform.

4. In all cases $\lim _{N \rightarrow \infty} d_{N}(i, j)=2 \log \frac{\pi}{2}, i \neq j \bmod$ $(N / 2)$. (See Fig. 1 for an illustration.)

Proof. To show that $\left(\mathcal{R}_{N}, d_{N}\right)$ is a semi-metric space we need to verify that (i) $d_{N}(i, i)=0$, (ii) $d_{N}(i, j)=d_{N}(j, i)$ and (iii) the triangle inequality holds. For a metric space we must further have (iv) $d_{N}(i, j) \neq 0$ unless $i=j$.

(i) is clearly satisfied as the projectors onto the eigenspaces are a resolution of the identity, $\sum_{k} \Pi_{k}=I$, and thus for any unit vector $|i\rangle$, we have $\left.\sum_{k=1}^{N}\left|\left\langle i\left|\Pi_{k}\right| i\right\rangle\right|=\sum_{k=1}^{N}\left|\Pi_{k}\right| i\right\rangle\left.\right|^{2}=$ 1. (ii) follows from $\left|\left\langle i\left|\Pi_{k}\right| j\right\rangle\right|=\left|\left\langle j\left|\Pi_{k}\right| i\right\rangle\right|$. The proof of the remaining properties relies on the circulant matrix property of the Hamiltonian $H_{1}$ in the first excitation subspace $\mathcal{H}_{1}$.

Specifically, $\left(H_{1}\right)_{i, i+1}=\left(H_{1}\right)_{i+1, i}=h$ for $i=$ $1, \ldots, N-1,\left(H_{1}\right)_{N, 1}=\left(H_{1}\right)_{1, N}=h$ and $\left(H_{1}\right)_{i, j}=0$ everywhere else. The eigenvalues and eigenvectors of $H_{1}$ are

$$
\begin{aligned}
& \lambda_{k}=h\left(\rho_{N}^{k}+\rho_{N}^{k(N-1)}\right)=2 h \cos \left(\frac{2 \pi k}{N}\right) \\
& w_{k}=\frac{1}{\sqrt{N}}\left(1, \rho_{N}^{k}, \rho_{N}^{k 2}, \ldots, \rho_{N}^{k(N-1)}\right)^{T}
\end{aligned}
$$

for $k=0, \ldots, N-1$, where $\rho_{N}^{k}:=e^{\frac{2 \pi \imath k}{N}}$ are $N$ th roots of unity. Observe the double eigenvalues $\lambda_{k}=\lambda_{N-k}$ except for $k=0$, and $N / 2$ if $N$ even. Thus, each of these double eigenvalues has two complex conjugate eigenvectors $v_{k}$ and $v_{k}^{*}$. These eigenvectors need not be orthogonal but observing that $\left\langle w_{k} \mid w_{\ell}\right\rangle=\delta_{k \ell}$ and $\left\langle w_{k} \mid w_{k}^{*}\right\rangle=0$, shows that

$$
\begin{aligned}
v_{0} & =w_{0}=\frac{1}{\sqrt{N}}(1,1, \ldots)^{T} \\
v_{k} & =w_{k}, \quad v_{N-k}=w_{k}^{*}, \quad k=1, \ldots N^{\prime}=\left\lfloor\frac{N-1}{2}\right\rfloor \\
v_{N / 2} & =w_{N / 2}=\frac{1}{\sqrt{N}}(1,-1, \ldots)^{T}, \quad \text { if } \mathrm{N} \text { is even }
\end{aligned}
$$

defines an orthonormal basis of $\mathcal{H}_{1}$. Furthermore, in the basis in which $H_{1}$ is circulant, $|i\rangle=e_{i}$, where $\left\{e_{i}: i=1, \ldots, N\right\}$ is the natural basis of $\mathbb{C}^{N}$. We have

$$
\begin{aligned}
\left|\left\langle i\left|\Pi_{0}\right| j\right\rangle\right| & =\left|\left\langle i \mid v_{0}\right\rangle\left\langle v_{0} \mid j\right\rangle\right|=\frac{1}{N} \\
\left|\left\langle i\left|\Pi_{k}\right| j\right\rangle\right| & =\left|\left\langle i \mid v_{k}\right\rangle\left\langle v_{k} \mid j\right\rangle+\left\langle i \mid v_{N-k}\right\rangle\left\langle v_{N-k} \mid j\right\rangle\right| \\
& =\left|\rho_{N}^{k i}\left(\rho_{N}^{k j}\right)^{*}+\left(\rho_{N}^{k i}\right)^{*} \rho_{N}^{k j}\right| \frac{1}{N} \\
& =\left|\rho_{N}^{k(i-j)}+\rho_{N}^{-k(i-j)}\right| \frac{1}{N}=\frac{2}{N}\left|\cos \left(\frac{2 \pi k(i-j)}{N}\right)\right| \\
\left|\left\langle i\left|\Pi_{N / 2}\right| j\right\rangle\right| & =\left|\left\langle i \mid v_{N / 2}\right\rangle\left\langle v_{N / 2} \mid j\right\rangle\right|=\frac{1}{N} .
\end{aligned}
$$


Summing over all eigenspaces $k=0, \ldots,\lfloor N / 2\rfloor$ gives

$$
\begin{aligned}
& \sqrt{p_{\max }(|i\rangle,|j\rangle)} \\
& =\left\{\begin{array}{c|c|c}
\frac{1}{N}+\frac{2}{N} \sum_{k=1}^{N^{\prime}}\left|\cos \left(\frac{2 \pi k(i-j)}{N}\right)\right|, & N=2 N^{\prime}+1 \\
\frac{2}{N}+\frac{2}{N} \sum_{k=1}^{N^{\prime}}\left|\cos \left(\frac{2 \pi k(i-j)}{N}\right)\right|, & N=2 N^{\prime}+2
\end{array}\right.
\end{aligned}
$$

For $i=j$ all cosines in Eq. (4) are equal to 1 and we have $\sqrt{p_{\max }(|i\rangle,|i\rangle)}=\left(1+2 N^{\prime}\right) / N=1$ for $N=2 N^{\prime}+1$ and $\sqrt{p_{\max }(|i\rangle,|i\rangle)}=\left(2+2 N^{\prime}\right) / N=1$ for $N=2 N^{\prime}+$ 2 , which shows that $d(i, i)=0$. For $N=2 N^{\prime}+1$ it is easy to see that $p_{\max }(i, j)=1$ if and only if $i=j$, hence (iv). For $N=2 N^{\prime}+2$, on the other hand, we also have $\left|\cos \left(\frac{2 \pi k N / 2}{N}\right)\right|=|\cos (\pi k)|=1$, and thus $d(i, j)=0$ for $i-j=N / 2$, i.e., the distance vanishes for antipodal points, and thus $d(i, j)$ is at most a semi-metric. However, noting that $d(i, j)=d\left(i, N^{\prime}+1+j\right)$ for $j \leq N^{\prime}+1$, we can identify antipodal points $|j\rangle$ and $\left|j+N^{\prime}+1\right\rangle$ and let $d$ be defined on the set of equivalence classes $[|j\rangle]$ for $j=1, \ldots, N^{\prime}+1$.

To show that the triangle inequality is satisfied, we show that $\sqrt{p_{\max }(|\ell\rangle,|m\rangle)} \sqrt{p_{\max }(|m\rangle,|n\rangle)} \leq \sqrt{p_{\max }(|\ell\rangle,|n\rangle)}$. From the definition of $p_{\max }$ in terms of the eigenvectors of $H_{1}$ we have

$$
\begin{aligned}
& \sqrt{p_{\max }(|\ell\rangle,|m\rangle)}=\frac{1}{N} \sum_{k=0}^{N-1} \alpha_{k} \rho_{N}^{k(m-\ell)} \\
& \sqrt{p_{\max }(|m\rangle,|n\rangle)}=\frac{1}{N} \sum_{k^{\prime}=0}^{N-1} \beta_{k^{\prime}} \rho_{N}^{k^{\prime}(n-m)}
\end{aligned}
$$

where $\alpha_{k}, \beta_{k^{\prime}}= \pm 1$. Setting

$$
\gamma_{k}=\sum_{k^{\prime}=0}^{N} \alpha_{k} \beta_{k^{\prime}} \rho_{N}^{\left(k^{\prime}-k\right)(n-m)}
$$

we obtain

$$
\begin{aligned}
& \sqrt{p_{\max }(|\ell\rangle,|m\rangle)} \sqrt{p_{\max }(|m\rangle,|n\rangle)} \\
= & \frac{1}{N^{2}} \sum_{k, k^{\prime}=0}^{N-1} \alpha_{k} \beta_{k^{\prime}} \rho_{N}^{k(m-\ell)} \rho_{N}^{k^{\prime}(n-m)} \\
= & \frac{1}{N^{2}} \sum_{k, k^{\prime}=0}^{N-1} \alpha_{k} \beta_{k^{\prime}} \rho_{N}^{k(n-\ell)+\left(k^{\prime}-k\right)(n-m)} \\
= & \frac{1}{N^{2}} \sum_{k=0}^{N-1} \gamma_{k} \rho_{N}^{k(n-\ell)}=\left|\frac{1}{N^{2}} \sum_{k=0}^{N-1} \gamma_{k} \rho_{N}^{k(n-\ell)}\right| .
\end{aligned}
$$

The final equality follows because the LHS and thus the RHS are known to be real and positive. Furthermore, as $\rho_{N}$ is a root of unity, $\left|\rho_{N}\right|=1$, and recalling $\left|\alpha_{k}\right|=\left|\beta_{k^{\prime}}\right|=1$,

$$
\begin{aligned}
\left|\gamma_{k}\right| & =\left|\rho_{N}^{k(m-n)} \sum_{k^{\prime}=0}^{N-1} \alpha_{k} \beta_{k^{\prime}} \rho_{N}^{k^{\prime}(n-m)}\right| \\
& \leq\left|\rho_{N}^{k(m-n)}\right| \cdot \sum_{k^{\prime}=0}^{N-1}\left|\alpha_{k} \beta_{k^{\prime}} \rho_{N}^{k^{\prime}(n-m)}\right|=N .
\end{aligned}
$$

Again we have $\rho_{N}^{(N-k)(m-n)}=\rho_{N}^{-k(m-n)}$, and as the LHS above is known to be real, we know that we must have $\gamma_{k}=$ $\gamma_{N-k}$. Hence, we can again collect exponential terms pairwise to obtain cosines, which gives for $N=2 N^{\prime}+1$ :

$$
\begin{aligned}
\left|\frac{1}{N^{2}} \sum_{k=0}^{N-1} \gamma_{k} \rho_{N}^{k(n-\ell)}\right| & =\left|\frac{\gamma_{0}}{N^{2}}+\frac{1}{N^{2}} \sum_{k=1}^{N^{\prime}} 2 \gamma_{k} \cos \left(\frac{2 \pi k(n-\ell)}{N}\right)\right| \\
& \leq \frac{\left|\gamma_{0}\right|}{N^{2}}+\frac{2}{N^{2}} \sum_{k=1}^{N^{\prime}}\left|\gamma_{k}\right|\left|\cos \left(\frac{2 \pi k(n-\ell)}{N}\right)\right| \\
& \leq \frac{1}{N}+\frac{2}{N} \sum_{k=1}^{N^{\prime}}\left|\cos \left(\frac{2 \pi k(n-\ell)}{N}\right)\right| \\
& =\sqrt{p_{\max }(|\ell\rangle,|n\rangle)}
\end{aligned}
$$

For $N=2 N^{\prime}+2$ we simply replace $\gamma_{0}$ by $\gamma_{0}+\gamma_{N^{\prime}+1}$ above to obtain

$$
\begin{aligned}
\left|\frac{1}{N^{2}} \sum_{k=0}^{N-1} \gamma_{k} \rho_{N}^{k(n-\ell)}\right| & \leq \frac{2}{N}+\frac{2}{N} \sum_{k=1}^{N^{\prime}}\left|\cos \left(\frac{2 \pi k(n-\ell)}{N}\right)\right| \\
& =\sqrt{p_{\max }(|\ell\rangle,|n\rangle)} .
\end{aligned}
$$

This proves (iii) and hence parts (1) and (2) of the theorem.

To establish (3) we note that if $N=2 N^{\prime}+1$ is prime then

$$
\sum_{k=1}^{N^{\prime}}\left|\cos \left(\frac{2 \pi k(i-j)}{N}\right)\right|=\sum_{k=1}^{N^{\prime}}\left|\cos \left(\frac{2 \pi k}{N}\right)\right|
$$

If $N$ is not $p$ or $2 p$ then $N$ and $(i-j)$ will have factors (which can be canceled) in common for some $(i-j)$ but not for others and hence we will obtain different distances.

To establish (iv) letting $N \rightarrow \infty$, it is easily seen that the dependency on $i, j$ is eliminated provided $i \neq j \bmod (N / 2)$. Hence, taking the norm of the above and then $-\log (\cdot)$ it follows that, for infinite rings, the distance is uniform for $i \neq j+\bmod (N / 2)$. Finally,

$$
\begin{aligned}
\lim _{N \rightarrow \infty} \sqrt{p_{\max }(|i\rangle,|j\rangle)} & =\lim _{N \rightarrow \infty} \frac{2}{N} \sum_{k=0}^{N / 2}|\cos ((i-j) 2 \pi k / N)| \\
& =\frac{4|i-j|}{2 \pi} \int_{0}^{\frac{\pi}{2|i-j|}} \cos (|i-j| x) d x \\
& =\frac{2|i-j|}{\pi|i-j|}[\sin (|i-j| x)]_{0}^{\frac{\pi}{2|i-j|}}=\frac{2}{\pi}
\end{aligned}
$$


shows that the limiting value for the distance is $d_{\infty}(i, j)=$ $-2 \log \frac{\pi}{2} \approx 2 \times 0.4516$ for $i \neq j \bmod (N / 2)$.

The preceding theorem points to discrete spaces which, possibly after some identification, become complete order $n$ graphs $K_{n}$ with uniform or nearly uniform link weight. Complete graphs with uniform link weight are among the very few that are embeddable in all spaces of constant curvature: negatively curved Riemannian manifolds $\mathbb{H}_{\kappa<0}$, Euclidean spaces $\mathbb{E}$, and positively curved Riemannian manifolds $\mathbb{S}_{\kappa>0}$. The latter embedding appears the most natural, since the various vertices are nearly uniformly "filling" the whole sphere, whereas it is impossible to uniformly fill a Euclidean or a hyperbolic space with finitely many vertices, as $\mathbb{S}_{\kappa}$ and $\mathbb{H}_{\kappa}$ are infinite. Also $K_{n}$ can be embedded in a sphere of dimension $(n-2)$, whereas the smallest dimension in which a nonpositively curved manifold can contain $n$ equidistant points is $(n-1)$. This not to say that it is futile to consider embeddings in, say, hyperbolic spaces; indeed, hyperbolic spaces have specific transport phenomena that could map to the graphs they support but we begin with embeddability in uniformly positively curved spaces.

The following theorem makes this embeddability precise. For notational convenience, let $\widetilde{\mathcal{R}}_{N}$ denote the ring $\mathcal{R}_{N}$ after anti-podal identification. Also define the following function

$$
\kappa_{\max }(n, w):=\left[w^{-1} \cos ^{-1}\left(-\frac{1}{n-1}\right)\right]^{2}
$$

where $w$ is typically some edge weight.

Theorem 2 The spin rings are embeddable in the following spaces:

1. If $N$ is an odd composite number, $\left(\mathcal{R}_{N}, d_{N}\right)$ is isometric to a complete graph $K_{N}$ with nonuniform link weight; furthermore, for $N$ large enough, there exists an isometric embedding $\left(\mathcal{R}_{N}, d_{N}\right) \hookrightarrow\left(\mathbb{S}_{\kappa}^{N-1}, d_{\kappa}\right)$ for $\kappa \leq \kappa_{\max }(N, \bar{w})$.

2. If $N=2 c$ where $c$ is a composite number, $\left(\widetilde{\mathcal{R}}_{N}, d_{N}\right)$ is isometric to a complete graph $K_{N / 2}$ with nonuniform link weight; furthermore, for $N$ large enough, there exists an isometric embedding $\left(\mathcal{R}_{N}, d_{N}\right) \hookrightarrow$ $\left(\mathbb{S}_{\kappa}^{(N / 2)-1}, d_{\kappa}\right)$ for $\kappa \leq \kappa_{\max }(N / 2, \bar{w})$.

3. a. If $N=p$, where $p$ is a prime number, $\left(\mathcal{R}_{N}, d_{N}\right)$ is isometric to a complete graph $K_{N}$ of uniform edge weight $w=d_{N}(i, j), i \neq j$; furthermore, there exists an isometric embedding $\left(\mathcal{R}_{N}, d_{N}\right) \hookrightarrow\left(\mathbb{S}_{\kappa}^{N-1}, d_{\kappa}\right)$ in the $(N-1)$-dimensional sphere of curvature $\kappa \leq$ $\kappa_{\max }(N, w)$. Finally, there exists an irreducible isometric embedding $\left(\mathcal{R}_{N}, d_{N}\right) \hookrightarrow\left(\mathbb{S}_{\kappa}^{N-2}, d_{\kappa}\right)$ in the sphere of curvature $\kappa=\kappa_{\max }(N, w)$.

b. If $N=2 p$ where $p$ prime, $\left(\widetilde{\mathcal{R}}_{N}, d_{N}\right)$ is isometric to a complete graph $K_{N / 2}$ with uniform link weight; furthermore, there exists an embedding $\left(\widetilde{\mathcal{R}}_{N}, d_{N}\right) \hookrightarrow$

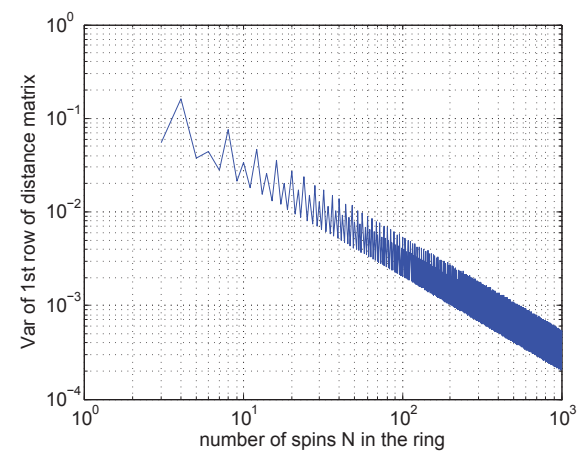

Fig. 1. Variance of quantum mechanical distance between spins showing its decrease as the number of spins increases.

$$
\begin{aligned}
& \left(\mathbb{S}^{N / 2-1}, d_{\kappa}\right) \text { for } \kappa \leq \kappa_{\max }(N / 2, w) \text { and an irre- } \\
& \text { ducible isometric embedding }\left(\widetilde{\mathcal{R}}_{N}, d_{N}\right) \hookrightarrow\left(\mathbb{S}^{N / 2-2}, d_{\kappa}\right) \\
& \text { for } \kappa=\kappa_{\max }(N / 2, d) \text {. }
\end{aligned}
$$

Proof. Part 3.a of the proof is in the Appendix.

Part 3.b of the proof is a corollary of the Appendix. By doing the anti-podal identification on the semi-metric space $\left(\mathcal{R}_{N}, d_{N}\right)$, one obtains the metric space $\left(\widetilde{\mathcal{R}}_{N}, d_{N}\right)$. The latter is clearly isomorphic to $\left(K_{N / 2}, w\right)$, where $w$ is the uniform link weight. The result then follows by applying the Appendix to $\left(K_{N / 2}, w\right)$.

Part 1 relies on the continuity of the Gram matrix relative to distance data. Define $\left(K_{\infty}, w_{\infty}\right)$ be the complete graph on countably infinitely many vertices with uniform link weight $w_{\infty}:=\lim _{N \rightarrow \infty} d_{N}(i, j), i \neq j$. By a limiting argument on the Appendix, it follows that $\left(K_{\infty}, w_{\infty}\right)$ is isometrically embeddable in an infinite-dimensional sphere (itself embedded in the Hilbert space $\ell^{2}$ as $\sum_{i=1}^{\infty} x_{i}^{2}=1 / \sqrt{\kappa}$ ) of curvature $\kappa \leq \kappa_{\max }\left(\infty, w_{\infty}\right)=\pi^{2} / 4 w_{\infty}^{2}$. Hence the associated Gram matrix $G_{\kappa}\left(D_{\infty}(1,2,3, \ldots)\right)$ for uniform link weight is positive definite. Trivially, the $N \times$ $N$ section of the Gram matrix $G_{\kappa}\left(D_{\infty}(1,2, \ldots)\right)_{N \times N}$ for uniform link weight is also positive definite. Since $\lim _{N \rightarrow \infty} G_{\kappa}\left(D_{N}(1, \ldots, N)\right)=G_{\kappa}\left(D_{\infty}(1,2, \ldots)\right)_{N \times N}$, and since the latter is positive definite, there exists a $N$ large enough such that $G_{\kappa}\left(D_{N}(1, \ldots, N)\right)>0$. The latter means that the ring $\mathcal{R}_{N}$ is isometrically embeddable in $\mathbb{S}_{\kappa}^{N-1}$.

Part 2 follows from a combination of the argument of Part 3.b and Part 1.

\section{CONCLUSION}

In conclusion, besides their 1-dimensional physical geometry, quantum rings have been shown to have higher dimensional geometry for the quantum mechanical distance. In forthcoming work will investigate how the geometry can be changed to improve transmission fidelities by local control. 


\section{APPENDIX: EMBEDDABILITY OF COMPLETE GRAPH IN CONSTANT CURVATURE SPACES}

Given a set $\mathcal{V}$ of $n$ vertices and the distance data $D(1,2, \ldots$, $n)=\{d(i, j)\}_{i, j=1, \ldots, n}$, isometric embedding of $(\mathcal{V}, D)$ in a Riemannian manifold of uniform curvature $\kappa \neq 0$ involves the Gram matrix $G_{\kappa}(D(1, \ldots, n)$ ) (we sometimes simplify this to $G_{\kappa}(D)$ ). In the positive curvature case, $G_{\kappa>0}(D)=\{\cos \sqrt{\kappa} d(i, j)\}_{i, j=1, \ldots, n}$ and in the negative curvature case $G_{\kappa<0}(D)=\{\cosh \sqrt{-\kappa} d(i, j)\}_{i, j=1, \ldots, n}$. $(\mathcal{V}, D)$ is embeddable in $\mathbb{S}_{\kappa}^{N-1}$ iff $\kappa \leq \frac{\pi^{2}}{\max _{i, j} d^{2}(i, j)}$ and $G_{\kappa}(D)>0\left(\right.$ see [2]). $G_{\kappa}(D)>0$ is of course equivalent to $\operatorname{det} G_{\kappa}(D(1, \ldots, k))>0, \forall k=1, \ldots, n$. $(\mathcal{V}, D)$ is embeddable in $\mathbb{H}_{\kappa<0}^{n-1}$ iff sign $\operatorname{det} G_{\kappa}(D(1, \ldots, k))=(-1)^{k+1}$ (see [2]).

Embeddability in Euclidean space involves the CayleyMenger matrix $C M(D(1, \ldots, n))=$

$$
\left(\begin{array}{ccccc}
0 & 1 & 1 & \ldots & 1 \\
1 & 0 & d(1,2)^{2} & \ldots & d(1, n)^{2} \\
1 & d(2,1)^{2} & 0 & \ldots & d(2, n)^{2} \\
\vdots & \vdots & \vdots & \ddots & \vdots \\
1 & d(n, 1)^{2} & d(n, 2)^{2} & \ldots & 0
\end{array}\right) .
$$

Embeddability in Euclidean space is equivalent to sign $\operatorname{det} C M(D(1, \ldots, k))=(-1)^{k}, k=2, \ldots, n$ (see [2]).

Embeddability of the complete graph with uniform link weight in both the constant curvature hyperbolic space and the constant curvature spherical space involves the special $k \times k$ Toeplitz structure

$$
T_{k}=\left(\begin{array}{cccc}
1 & c & \ldots & c \\
c & 1 & \ldots & c \\
\vdots & \vdots & \ddots & \vdots \\
c & c & \ldots & 1
\end{array}\right), \quad k \geq 1
$$

where $c=\cosh (d(i, j) \sqrt{-\kappa})$ in the hyperbolic case and $c=\cos (d(i, j) \sqrt{\kappa})$ in the spherical case. The issue is the sequence of principal minors of such a Toeplitz-structured matrix. Set $t_{k}=\operatorname{det} T_{k \times k}$ and we have the following lemma:

Lemma 1 The recursion on the principal minors of the Toeplitz-structured matrix $T_{k}$ is

$$
t_{k+1}=(1-c) t_{k}+(1-c)^{2} t_{k-1}-(1-c)^{3} t_{k-2}
$$

subject to the initial conditions

$$
\begin{aligned}
& t_{1}=1 \\
& t_{2}=1-c^{2} \\
& t_{3}=(1-c)^{2}(2 c+1) .
\end{aligned}
$$

Furthermore, the solution to the above recursion is given by

$$
t_{k}=(1-c)^{k-1}((k-1) c+1), \quad k \geq 1 .
$$

Proof. By subtracting the first column from the last column of $T_{k}$, we get

$$
\operatorname{det} T_{k}=(-1)^{k+1}(c-1) \operatorname{det} T+(1-c) \operatorname{det} T_{k-1}
$$

where $T$ is the Toeplitz matrix with 1's on the superdiagonal and $c$ 's everywhere else. Again, by subtracting the first row from the last row of $T$, we get

$$
\operatorname{det} T=(-1)^{k+1}(c-1) \operatorname{det}\left(\begin{array}{cc}
c & c 1_{k-3}^{T} \\
c 1_{k-3} & T_{k-3}
\end{array}\right)
$$

where $1_{k}$ is the $k$-dimensional column made up of 1 's. Observing that

$$
\left(\begin{array}{cc}
c & c 1_{k-3}^{T} \\
c 1_{k-3} & T_{k-3}
\end{array}\right)=\left(\begin{array}{cc}
(c-1) & 0 \\
0 & 0
\end{array}\right)+T_{k-2}
$$

and remembering that the determinant of the sum of two matrices equals the sum of the determinants of all matrices constructed with some columns of the first matrix and the complementary columns of the second matrix, we get

$$
\begin{aligned}
\operatorname{det}\left(\begin{array}{cc}
c & c 1_{k-3}^{T} \\
c 1_{k-3} & T_{k-3}
\end{array}\right) & =\operatorname{det} T_{k-2}+\operatorname{det}\left(\begin{array}{cc}
(c-1) & c 1_{k-3} \\
0 & T_{k-3}
\end{array}\right) \\
& =\operatorname{det} T_{k-2}+(c-1) \operatorname{det} T_{k-3} .
\end{aligned}
$$

Combining all of the above yields the recursion. The initial conditions on the recursion are trivial to verify. The explicit solution is easily seen by direct verification to satisfy the recursion and its initial conditions.

From the above, it is possible to say something about the eigenvalues of $T_{n}$.

\section{Corollary 1}

$$
\operatorname{det}\left(s I-T_{n}\right)=(s-(1-c))^{n-1}(s-((n-1) c+1)) .
$$

Proof. Recall that the coefficient of $s^{n-k}$ in $\operatorname{det}\left(s I-T_{n}\right)$ is $(-1)^{k}$ times the sum of all principal minors of order $k$ of $T_{n}$. There are $\left(\begin{array}{l}n \\ k\end{array}\right)$ such principal minors, all equal to $t_{k}$. Hence,

$$
\begin{aligned}
\operatorname{det} & \left(s I-T_{n}\right)=\sum_{k=0}^{n}(-1)^{k}\left(\begin{array}{l}
n \\
k
\end{array}\right) t_{k} s^{n-k} \\
= & \sum_{k=0}^{n}(-1)^{k}\left(\begin{array}{l}
n \\
k
\end{array}\right)(1-c)^{k-1}((k-1) c+1) s^{n-k} \\
= & \left(\sum_{k=0}^{n-1}(-1)^{k}\left(\begin{array}{c}
n-1 \\
k
\end{array}\right)(1-c)^{k} s^{n-1-k}\right) \\
= & \quad(s-(1-c))^{n-1}(s-((n-1) c+1))
\end{aligned}
$$

We now look at the recursion in the case of the CayleyMenger matrix. 
Lemma 2 The recursion on the $k \times k$ top left-hand corner principal minors $\mathrm{cm}_{k}:=\operatorname{det}\left(C M_{k \times k}\right)$ of the CayleyMenger matrix for uniform distance $d$ is given by

$$
\mathrm{cm}_{k}=-\left(\frac{(k-1)}{d^{2}(k-2)}\right) t_{k-1}
$$

where the recursion on $t_{k-1}$ is

$$
t_{k-1}=-\left(\frac{(k-2) d^{2}}{k-3}\right) t_{k-2}
$$

Proof. Applying the Schur lemma to

$$
\mathrm{CM}_{k \times k}=\left(\begin{array}{cc}
0 & 1_{k-1}^{T} \\
1_{k-1} & T_{k-1}
\end{array}\right)
$$

where $T_{k-1}$ is the Toeplitz matrix with 0's on the diagonal and $d^{2}$ 's everywhere else, we get

$$
\mathrm{cm}_{k}=-\left(1_{k-1}^{T} T_{k-1}^{-1} 1_{k-1}\right) \operatorname{det}\left(T_{k-1}\right) .
$$

If we now observe that $1_{k-1}$ is an eigenvector of $T_{k-1}$ with eigenvalue $d^{2}(k-2)$, it follows that $1_{k-1}^{T} T_{k-1}^{-1} 1_{k-1}=(k-$ 1) $/\left(d^{2}(k-2)\right)$ and setting $t_{k-1}:=\operatorname{det}\left(T_{k-1}\right)$ the first part of the recursion follows. Next, if we apply exactly the same Schur lemma argument to

$$
T_{k-1}=\left(\begin{array}{cc}
0 & d^{2} 1_{k-2}^{T} \\
d^{2} 1_{k-2} & T_{k-2}
\end{array}\right)
$$

the second part of the recursion follows.

Proposition 1 The complete graph $K_{n \geq 2}$ with uniform link weight $d(i, j)>0, i \neq j$, is irreducibly isometrically embeddable in $\mathbb{E}^{n-1}$ and in $\mathbb{H}_{\kappa<0}^{n-1}$. The same graph is isometrically embeddable in $\mathbb{S}_{\kappa>0}^{n-1}$ iff

$$
\kappa \leq\left[d(i, j)^{-1} \cos ^{-1}\left(-\frac{1}{n-1}\right)\right]^{2} .
$$

Furthermore, it is irreducibly isometrically embeddable in $\mathbb{S}_{\kappa>0}^{n-2}$ for

$$
\kappa=\left[d(i, j)^{-1} \cos ^{-1}\left(-\frac{1}{n-1}\right)\right]^{2} .
$$

Proof. The proof of Euclidean embedding follows at once from Lemma 2, as the latter indeed reveals that sign $\operatorname{det}\left(\mathrm{CM}_{k \times k}\right)$ is alternating.

For embeddability in hyperbolic space, set $c=\cosh (d(i, j) \sqrt{-\kappa})>1$, and then the lemma yields $\operatorname{det} G_{\kappa}(D(1, \ldots, k))=(1-c)^{k-1}((k-1) c+1)$. The principal minors of $G_{\kappa}(D(1, \ldots, n))$ clearly never vanish and their signs have the required alternating property, from which irreducible isometric embedding follows.

For embeddability in spherical space, set $c=\cos (d(i, j) \sqrt{\kappa}) \leq 1$ and then the lemma yields
$G_{\kappa}(D(1, \ldots, k))=(1-c)^{k-1}((k-1) c+1)$. Isometric embeddability is hence equivalent to the sequence $(k-1) c+1$, $k=1, \ldots, n$ being positive, with possibly a vanishing tail. $(k-1) c+1 \geq 0$ is clearly equivalent to

$$
\kappa \leq\left[d(i, j)^{-1} \cos ^{-1}\left(-\frac{1}{k-1}\right)\right]^{2}
$$

and since

$$
\cos ^{-1}\left(-\frac{1}{n-1}\right)<\cos ^{-1}\left(-\frac{1}{k-1}\right), \quad k<n
$$

it follows that $\operatorname{det} G_{\kappa}(D(1, \ldots, k))=(1-c)^{k-1}((k-1) c+$ 1 ) could only possibly vanish for $k=n$ and is positive for $k<n$. Hence the graph is isometrically embeddable iff (7) is satisfied $\forall k \leq n$, which is equivalent to 5 . The irreducible isometric embedding in $\mathbb{S}_{\kappa}^{n-2}$ requires, in addition, that $(n-$ 1) $c+1=0$, which is equivalent to 6 .

\section{REFERENCES}

[1] F. Ariaei, M. Lou, E. Jonckheere, B. Krishnamachari, and M. Zuniga, Curvature of indoor sensor network: clustering coefficient, J. Wireless Communications and Networking, vol. 2008, Article ID 213185, 2008.

[2] L. M. Blumenthal, Theory and Applications of Distance Geometry, Oxford Clarendon Press, London, 1953.

[3] M. R. Bridson and A. Haefliger, Metric Spaces of NonPositive Curvature, vol. 319 of A Series of Comprehensive Surveys in Mathematics, Springer, New York, 1999.

[4] M. Gromov, Hyperbolic groups, in Essays in Group Theory, S. M. Gersten, Ed., vol. 8 of Mathematical Sciences Research Institute Publication, pp. 75-263. Springer, New York, 1987.

[5] M. Gromov, Metric Structures for Riemannian and NonRiemannian Spaces, vol. 152 of Progress in Mathematics, Springer, New York, 2001.

[6] E. Jonckheere, P. Lohsoonthorn, and F. Bonahon, Scaled Gromov hyperbolic graphs, J. Graph Theory, vol. 57, pp. 157-180, 2008.

[7] E. Jonckheere, F. Ariaei, and P. Lohsoonthorn, Scaled Gromov four-point condition for network graph curvature computation, Internet Mathematics, vol. 7, no. 3, pp. 137-177, 2011.

[8] E. Jonckheere, S. Schirmer, and F. Langbein, Geometry and curvature of spin networks, in IEEE MultiConference on Systems and Control, pp. 786-791, 2011. (Available at arXiv:1102.3208v1.)

[9] M. Kaku, Introduction to Superstrings, Graduate Texts in Contemporary Physics. Springer, New York, 1998. 
[10] P. Lohsoonthorn, E. Jonckheere, and F. Ariaei, Upper bound on scaled Gromov-hyperblic delta, J. App. Mathematics and Computation, vol. 192, pp. 191-204, 2007.

[11] N. Prakash, Mathematical Perspectives on Theoretical Physics: A Journey from Black Holes to Superstrings, Tata McGraw-Hill, New Delhi, 2000. 